\title{
A New Approach for Solving the Unit Commitment Problem by Cuckoo Search Algorithm
}

\author{
Alireza Gharegozi* and Rozbeh Jahani \\ Shahindezh Branch, Islamic Azad University, Shahindezh, Iran; gharegozi@yahoo.com
}

\begin{abstract}
In this paper, a new approach to solving the issue of optimal production planning (Unit Commitment or UC) has been presented by using Cuckoo Search Algorithm. Results of testing this paper indicate that not only this algorithm includes all limits of issue well, but it has also the benefits all such as the most appropriate convergence in the response, high computational speed and high accuracy. A sample power system with 10 power plant units has been used in this study in order to test this new algorithm. Optimal planning in generation of power units of this system is also obtained from the results of experiment and they will be inferable given the establishment and observation of the constraints in the target function of issue. Numerical results of this paper indicate that the performance of Cuckoo Search Algorithm has had significant improvement than other algorithms and it results in the minimum production cost.
\end{abstract}

Keywords: Cuckoo Search Algorithm, Power Systems, Optimal Production Planning, System Constraints, System Limitations.

\section{Signs and Abbreviations}

i: Specifies the unit.

t: Timeframes.

G: Number of generator units.

$U_{i t}$ : Status of on or off $i$ unit in the timeframe t.

$P_{i t}$ : Generation power of unit $\mathrm{i}$ in the timeframe $\mathrm{t}$.

$F_{i}\left(P_{i t}\right)$ : Current cost function of power plant unit i in the timeframe $\mathrm{t}$.

$a_{i}-b_{i}-c_{i}$ : Coefficients of cost function i.

$S_{i}$ : cost of starting up the unit i.

$\tau_{i}-S_{1 i}-S_{0 i}$ : Coefficients of startup cost for unit i.

$P_{D t}$ : System load demand in the timeframe t.

$\lambda$ : Rate of increased loss cost.

$P_{\text {imax }}$ : Maximum production capacity of unit i.

$P_{\text {imin }}:$ Minimum production capacity of unit i.

$M_{i}$ : Time constraints in turning on or off the unit i.

$\mathrm{TO}_{i}$ : Minimum startup time for unit i.
TS : Minimum time of turning off for unit i.

$T O_{i}$ : The length of time during which the unit is on.

$T S_{i}$ : The length of time during which the unit is off.

\section{Introduction}

The purpose of economic planning of power units is to achieve an optimal combination of producing units connected to the network in order to supply the load of network. The issue of economic load distribution is classified into two sub-problems: 1) UC problem, 2) Online load economic distribution (optimal load distribution). The UC problem selects the units which supply the predicted load of system at the lowest cost in a specified timeframe and also includes spinning reserve requirements. Sub-issue of load economic distribution distributes the predicted load in the selected power plant units so that the network load is distributed among the generating units in a way that

* Corresponding author:

Alireza Gharegozi (gharegozi@yahoo.com) 
the total cost becomes totally minimized. If all units in a system seek to enter the circuit simultaneously in order to provide the load, it will be costly and result in the high cost for the system; hence, an optimal combination of units should be selected to supply this load (in a specified timeframe). This will save a considerable cost. UC problem can be formulated in a way that the cost function is minimized, thus the operating cost function is minimized, so the minimized turned on and off time, minimized power constraints, production constraints, and spinning reserve and... are estimated [1]. The exact solution in the UC problem can be obtained by the complete numerical methods such as integer or dynamic programming. One of the disadvantages of these methods is the large volume of computations and thus the increased computation time which is enhanced by increasing the number of units exponentially and will have the high memory. Improved mentioned conventional methods can increase the accuracy of solution in a reasonable and appropriate time [2]. UC problem model can be considered for the large-scale power systems while resulted in a reasonable time in the calculations. Various numerical optimization techniques can be used for solving the UC problem; some of these methods are: priority list method [3] and [4], integer programming [5] and [6], dynamic programming [7] and [8], mixed integer programming [9], and Branch-and-Bound (B \& B) Method [10] and the method of Lagrange multipliers [11] and [12]. Among these methods: Priority list Method is simple and fast, but there is no guarantee of its output response quality and convergence. Dynamic programming methods, which are based on the Priority list, are flexible, but they are costly in terms of calculations. $\mathrm{B} \& \mathrm{~B}$ Method uses the linear functions for representing the cost of fuel consumption and (time-dependent) operating cost, but one of the disadvantages of this method is the exponential growth while running the program which is consistent with the dimensions of system. Methods for integer and integer-combined programming followed the linear programming techniques in solving and reaching an integer response. These methods are used for small dimensions of UC and require the multiple assumptions which restrict the solution space. Lagrange multipliers method will result in a quick solution, but may be faced with the problem in the response convergence. Long with the above mentioned methods, there is another group of numerical techniques which can be efficient in solving the UC problem. Some of these techniques include: Artificial Neural
Networks [13] and [14], simulation based on melting the metals (SA) [15] and genetic algorithm (GA) [16]. These methods can be adapted to the complex constraints of systems and it is claimed that they offer better quality responses. The method (SA) is a powerful method based on the estimated optimization techniques which will have the desirable convergence theoretically. (Coefficient of convergence possibility is equal to 1 ). The increased time of CPU in finding the optimal point is one of the disadvantages of this method. The genetic algorithm methods are the estimated methods which are based on the natural selection and genetics and work based on the parallel search. In this paper, a new method called Cuckoo Search Algorithm has been used for solving UC problem.

\section{Formulating the UC Problem}

\subsection{Target Function}

In this paper, it is assumed that the planned timeframe is 24 hours and is divided into 24 one-hour timeframes. Total cost is the sum of the current costs (fuel) and startup cost for the entire units in all scheduled intervals. According to this fact, the target function of UC problem is defined as follows:

$$
\operatorname{MinF}\left(U_{i t}, P_{i t}\right)=\sum_{t=1}^{24} \sum_{i=1}^{G}\left[U_{i t} F_{i t}\left(P_{i t}\right)+U_{i t}\left(1-U_{i t-1}\right) S_{i}\right]
$$

In general, the function of current costs (fuel cost) which is given as per unit in each timeframe, is a function of output power of power plant units. This function is discussed as follows:

$$
F_{i}\left(P_{i t}\right)=a_{i} P_{i t}^{2}+b_{i} P_{i t}+c_{i}
$$

The startup cost per unit depends on the off time before starting. This time-dependent starting up cost can be expressed according to the following formula:

$$
S_{i}=S_{0 i}+S_{1 i}\left(1-e^{-T / \tau_{i}}\right)
$$

\subsection{System Constraints}

Numerous constraints can be applied in the UC problem. Each network and power system can apply various provisions in the programming of units. The constraints depend on the arrangement of generators, features of load curve and so on. Spinning reserve is also defined 
as the total production capacity of all generators in the system subtracted from the existing load in the system and the possible losses imposed to the system. Spinning reserve should be provided in a way that the losses of one or more units create the security at the system frequency. Spinning reserve should be able to offset the losses in very heavy loads of units. In a specified time period, the spinning reserve requirement is defined as a function of insufficient production probability for supplying the consumer load.

\subsubsection{Power Balance Constraint}

$$
\sum_{i=1}^{G} U_{i t} P_{i t}=P_{D t}, t=1,2,3, \ldots
$$

$P_{i t}$ is calculated from the load distributed in the timeframe $\mathrm{t}$ which is according to the rate of increasing the losses $(\lambda)$; we have:

$$
\frac{d F_{1 t}}{d P_{1 t}}=\frac{d F_{2 t}}{d P_{2 t}}=\ldots=\frac{d F_{i t}}{d P_{i t}}=\lambda
$$

$t=1,2, \ldots, 24, i=1,2, \ldots, G$

\subsubsection{Spinning Reserve}

If the spinning reserve more than $7 \%$ of the total load at each timeframe is essential, we will have:

$$
\sum_{i=1}^{G} U_{i t} P_{i \max } \geq 1.07 P_{D t}, t=1,2,3, \ldots 24
$$

\subsubsection{Range of Output Power of Power Plant Units}

$t=1,2, \ldots, 24, i=1,2, \ldots, G \quad P_{i \min } \leq P_{i} \leq P_{i \max }$

\subsubsection{Time Limit of Turning on and off}

$$
\sum_{i=1}^{24}\left|U_{i t}-U_{i t-1}\right| \leq M_{i}
$$

\subsubsection{Constraints of Minimum Time of Turning on} and off

$$
\begin{aligned}
& T O_{i} \geq \underline{T O_{i}} \\
& T S_{i} \geq \underline{T S_{i}}
\end{aligned}
$$

\section{An Overview of Cuckoo Search Algorithm}

Cuckoo Optimization Algorithm (COA) flowchart is shown in the Figure 1. Like other evolutionary algorithms, the COA begins with an initial population which consists of Cuckoos. This population of cuckoos has eggs which lay them into the host birds' nests. Some of these eggs, which are more similar to the host bird's eggs, have more chance to grow and become the young cuckoos. Other eggs are recognized and disappeared by the host bird. The rate of grown nest eggs shows the suitability of the nests in that area. The more eggs in the environment are able to be survived and saved, the more profit (tendency) is assigned to that area. Therefore, a situation, in which the greatest numbers of eggs are saved, is the parameter which the COA intends to optimize it.

Cuckoos search for the best place for maximizing the survival of their own eggs. The communities and groups are created after the cuckoos' chicks get out from the eggs and change into the adult cuckoos. Each group has its own habitat. The best habitat for all groups will be the cuckoos' next destination in other groups. All groups migrate to the best existing region. Each group resides in an area close to the current position. Several egg laying radii will be calculated and created by considering the number of egg, which each cuckoo will lay, in addition to the distance of cuckoos from the current optimal area for habitat.

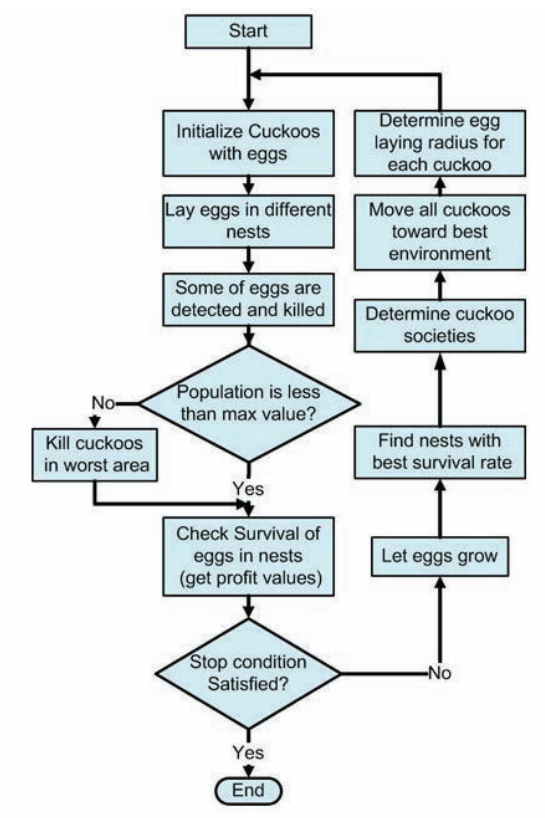

Figure 1. Cuckoo Optimization Algorithm (COA) flowchart. 


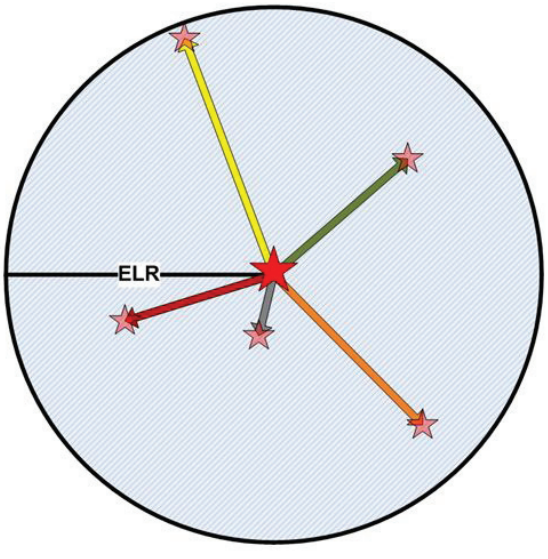

Figure 2. The Egg Laying Radius (ELR).

Then, the cuckoos start laying the eggs in the nests within their own egg laying radius. This process continues until reaching the best place for laying the egg (The region with the highest income). This optimal location is where the greatest numbers of cuckoos are gathered.

Building the cuckoos' primary habitat (initial population of candidate solutions):

For solving an optimal issue, it is necessary to form the variables of issue in the array form. These arrays are determined with the names "chromosome" and "particle position" in the GA and PSO, but this array is called the "habitat" in Cuckoo Optimization Algorithm (COA).

In an optimization problem, the next $\mathrm{N}_{\mathrm{var}}$ of a habitat will be a $1 \mathrm{xN}_{\mathrm{var}}$ array which shows the current position of cuckoos. This array is defined as follows:

$$
\text { Habitat }=\left[\mathrm{x} 1, \mathrm{x} 2, \ldots, \mathrm{x}_{\text {Nvar }}\right]
$$

The suitability amount (or benefit rate) in current habitat is calculated by evaluating the profit function $\left(f_{p}\right)$ in habitat. Thus:

Profit $=f_{p}($ habitat $)=f_{p}\left(x 1, x 2, \ldots, x_{\text {Nvar }}\right)$

As it can be seen, the COA is an algorithm which maximizes the profit function. For applying the COA in minimization problems, we should just multiply a negative sign by the cost function.

A habitat matrix with the size $\mathrm{N}_{\text {pop }}{ }^{*} \mathrm{~N}_{\text {var }}$ is built in order o start an optimization algorithm. A random numbers of eggs are assigned for each of these habitats. In the nature, each cuckoo lays 5 to 20 eggs. These numbers are used as the higher and lower limit for assignment of egg to each cuckoo in different iterations. Each cuckoo's another habit is that they are lay their eggs in a specific domain.
Hereinafter, the maximum egg-laying domain is called the Egg Laying Radius (ELR).

In an optimization problem, the higher limit of variable is shown by var $_{\text {hi }}$ and the lowest limit is shown by $\operatorname{var}_{\text {low }}$. Each cuckoo has ELR which is proportional to the total number of eggs, number of current eggs as well as the higher and lower limits of issue variables.

Therefore, ELR is defined as follows:

$E L R=\alpha \times \frac{\text { Number of current cuckoo's eggs }}{\text { Total number of eggs }} \times\left(\right.$ Var $_{\text {hi }}-$ Var $\left._{\text {low }}\right)$

Alpha is the variable by which we adjust the maximum value of ELR.

\section{Cuckoos' Method for Laying the Eggs}

Each cuckoo lays randomly the eggs in the host birds' nests which are in the ELR.

When all cuckoos lay their eggs, some of the eggs, which are less similar to the host bird's eggs, are identified and thrown out of the nest. Thus, after each egg-laying, $\mathrm{p} \%$ of all eggs (usually $10 \%$ ), which their profit function is lower, are eliminated. Remaining chicks feed and grow in the host nests.

Another interesting point about the cuckoo chicks is that only an egg can grow in each nest because when the cuckoo chicks get out of the eggs, they throw out the host bird's eggs from the nests and if the host chicks get out of the eggs sooner, the cuckoo chick eats the largest amount of food which the host bird brings (the chick pushes the others over with its 3 times larger body) and after a few days the host bird's chicks will die of starvation and only the cuckoo's chick survives.

\subsection{Cuckoos' Immigration}

When the cuckoos chicks are grown up and become young, they live in their own groups and environments, but when the time of laying the eggs becomes close, they will migrate to better habitats where the chance of eggs' survival is higher. After forming the cuckoo groups in different geographical areas (searching space of issue) the group with the best position is selected for other cuckoos as the target location for migration.

When the mature cuckoos live in all parts of environment, it is difficult to determine which cuckoo belongs to 


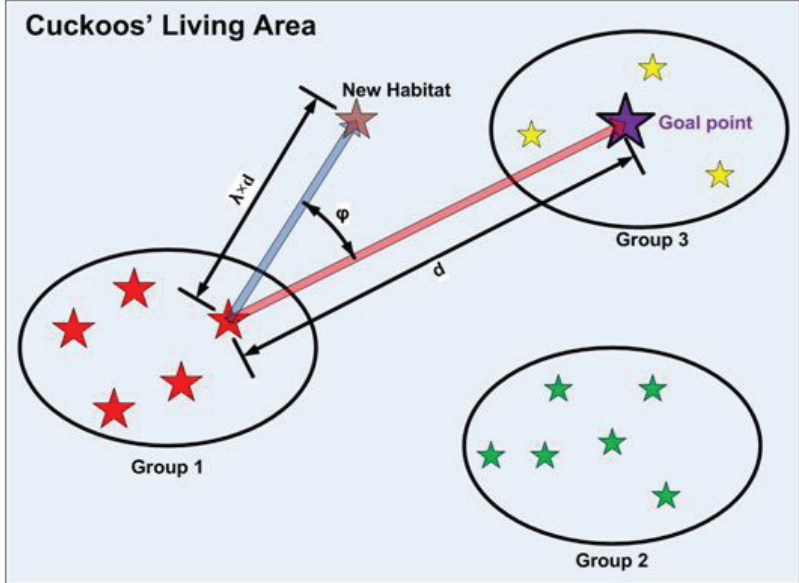

Figure 3. Cuckoos' Immigration.

which group. For solving this problem, the cuckoos' grouping is performed by classification method $\mathrm{K}$-means ( $\mathrm{a} \mathrm{k}$ from 3 to 5 is usually sufficient).

When the cuckoos' groups are made, the average profit of group is calculated in order to obtain the relative optimality of group habitat. Then, the group with the highest average profit (optimality) is selected as the target group and other groups migrate to it.

During the migration to the target point, the cuckoos do not pass all the way to the target point. They just pass a part of path in which they also have the deviation. Such this movement is clearly shown in the Figure 3.

As shown in the above figure, each cuckoo only passes $\lambda \%$ of the whole path towards the current ideal goal and it has also a deviation of $\varphi$ Radian. These two parameters help the cuckoo to seek further environment. $\lambda$ is a random number from 0 and 1 , and $\varphi$ is a number from $-\pi / 6$ to $\pi / 6$.

When all cuckoos migrated to the target location and the new residential areas of each one is determined, each cuckoo owns a large number of eggs. Given the number of each cuckoo's egg, an ELR is determined for it and then the egg-laying starts.

\subsection{Eliminating the Cuckoos in Inappropriate Areas}

Given this fact that there is always a balance for the population of birds in the nature, the number like $\mathrm{N}_{\max }$ controls and limits the maximum number of cuckoos who can live in an environment. This balance is established due to the dietary restrictions, hunted by the hunters and the inability to find the suitable nests for eggs.

\subsection{Convergence of the Algorithm}

After a few iterations, the whole cuckoo population reaches an optimal point with the maximal similarity of eggs to the host birds' eggs as well as the location of largest amount of food sources. This place will have the highest overall profit and the lowest number of eggs will be destroyed there. The convergence more than $95 \%$ for all cuckoos towards a point causes that the Cuckoo Optimization Algorithm (COA) to be ended.

\section{Optimization Through Cuckoo Search Algorithm}

Determining the on and off generators for a period of time (here 24 hours) is the issue of entering the power plants into the circuit, so that the cost of utilizing the generators of system becomes minimized. Constraints in this issue contain the following cases:

1. Minimum on and off time: After turning on, a generator cannot be turned off for a specified time and after turning off it cannot be remained on for a certain time. These constraints are considered as the $\mathrm{T}_{\text {on }}$ and $\mathrm{T}_{\text {off }}$ for generators.

2. The other constraint of system is the existence of reserve capacity in the system so that if one of the generators quits the circuit, the generators of systems will no longer be able to provide the network load. This constraint is as the spinning reserve capacity of model and indicates that the total capacity of maximum turned on units should be higher than the available load plus an overcapacity.

3. Other issues, which should be considered in this issue, include:

The overcapacity in the system should not exceed a certain limit, and in the case of existed overcapacity and the possibility to turn off the generator, these overcapacities should be eliminated.

The programming process has been as follows:

First, an initial population, which includes 5 suggestions for turning on and off the generators randomly, is generated. This initial population is shown with the variable ${ }_{\mathrm{INI}} \mathrm{POP}$.

After the random population generation, the sum of turned on (positive) numbers and turned off (negative) numbers should be 24 (in absolute value). 
Table 1. Optimal unit commitment result

\begin{tabular}{|c|c|c|c|c|c|c|c|c|c|c|c|c|c|c|c|c|c|c|c|c|c|c|c|c|}
\hline \multirow{2}{*}{$\begin{array}{l}\text { Unit } \\
\text { Number }\end{array}$} & \multicolumn{24}{|c|}{ On/Off Statue of Per Time-Step } \\
\hline & 1 & 2 & 3 & 4 & 5 & 6 & 7 & 8 & 9 & 10 & 11 & 12 & 13 & 14 & 15 & 16 & 17 & 18 & 19 & 20 & 21 & 22 & 23 & 24 \\
\hline 1 & 1 & 1 & 1 & 1 & 1 & 1 & 1 & 1 & 1 & 1 & 1 & 1 & 1 & 1 & 1 & 1 & 1 & 1 & 1 & 1 & 1 & 1 & 0 & 0 \\
\hline 2 & 1 & 1 & 1 & 1 & 1 & 1 & 1 & 1 & 1 & 1 & 1 & 1 & 1 & 1 & 1 & 1 & 1 & 1 & 1 & 1 & 1 & 1 & 0 & 0 \\
\hline 3 & 0 & 0 & 0 & 0 & 0 & 1 & 1 & 1 & 1 & 1 & 1 & 1 & 1 & 1 & 1 & 1 & 1 & 1 & 1 & 1 & 1 & 1 & 1 & 0 \\
\hline 4 & 0 & 0 & 0 & 0 & 0 & 0 & 0 & 0 & 0 & 0 & 0 & 0 & 0 & 0 & 1 & 1 & 1 & 1 & 1 & 0 & 0 & 0 & 0 & 0 \\
\hline 5 & 0 & 0 & 0 & 0 & 0 & 0 & 0 & 0 & 0 & 1 & 1 & 1 & 1 & 1 & 1 & 1 & 1 & 1 & 0 & 0 & 0 & 0 & 0 & 0 \\
\hline 6 & 0 & 0 & 0 & 0 & 0 & 0 & 0 & 1 & 1 & 1 & 1 & 1 & 1 & 1 & 0 & 0 & 0 & 0 & 0 & 0 & 0 & 1 & 1 & 1 \\
\hline 7 & 0 & 0 & 0 & 0 & 0 & 0 & 0 & 0 & 1 & 1 & 1 & 1 & 1 & 1 & 1 & 0 & 0 & 0 & 0 & 0 & 0 & 1 & 1 & 1 \\
\hline 8 & 0 & 0 & 0 & 0 & 1 & 1 & 1 & 1 & 1 & 1 & 1 & 1 & 1 & 1 & 1 & 1 & 1 & 1 & 1 & 1 & 1 & 1 & 1 & 0 \\
\hline 9 & 0 & 0 & 0 & 0 & 0 & 0 & 0 & 0 & 0 & 0 & 0 & 0 & 0 & 0 & 0 & 0 & 0 & 0 & 0 & 0 & 0 & 0 & 0 & 1 \\
\hline 10 & 0 & 0 & 0 & 0 & 0 & 0 & 0 & 0 & 0 & 0 & 0 & 0 & 1 & 1 & 1 & 1 & 1 & 1 & 1 & 1 & 1 & 0 & 1 & 0 \\
\hline
\end{tabular}

Table 2. Comparison results of various algorithms

\begin{tabular}{lc}
\hline Algorithms & Total Cost \\
\hline LR [18] & 80766.0 \\
Hopfield-SA [19] & 79114.6 \\
Evolutionary Method [20] & 79043 \\
AC-PSO[21] & 79010.1 \\
HPSO [22] & 81118.3 \\
GA[23] & 78988.8 \\
GA [24] & 79807.0 \\
COA & 78993.2 \\
\hline
\end{tabular}

After generating this initial population in the units, the following constraints should be modified in the units:

1. The units should be turned on equal to the minimum turned on time and to be turned off equal to the minimum turned off time (mdt), thus the numbers are modified in a way that this constraints are met.

2. Since by above modification the total on and off hours becomes more than 24 hours, this time should reach 24 hours by observing the mentioned constraints.

3. Then these numbers, which are integer, are converted to binary numbers for each time so that 1 indicates On and zero indicates turned Off unit.

4. Then given the spinning reserve constraint, the units become turned on respectively for each hour in the hours in which the number of units does not provide the spinning capacity.

5. We send out the additional units from the circuit, if possible, by considering the constraint related to the minimum on or off time because the overcapacity may be created in the system by performing this stage.

6. After that, the economic load distribution is performed on the initial population in order to determine the populations with the least cost.

Note: The initial population is considered equal to 6 and the $6^{\text {th }}$ direction has been considered as the suggestion for the optimal hypothetical point.

By generating the initial population, we enter into the optimization with Cuckoo Search Algorithm.

In this part of cell array, CUCKOO POP has been applied for generating the population of egg numbers and status and location of each egg.

In the first case, between 2 to 4 eggs are produced randomly for each population and they are indicated by the cell array CukooPop. number Of Eggs.

Then the egg drop radius is calculated with respect to this number of eggs which are produced for the initial population and the egg-laying starts (i.e. the egg is produced for each population by adding the random numbers of new population).

Thereupon, the constraints, which were observed in the initial population, are observed for these new populations. The eggs and populations are created by performing this stage.

Populations are converted into binary numbers.

The cost of each population and eggs are calculated by doing the economic load distribution by Lambda Iteration method.

Given that the cost of starting up should be considered as warm and cold costs, these costs are also added to the operation cost. 
Then given that the maximum 10 populations per iteration can be remained, the populations are classified based on their costs and the units with higher costs are removed.

Thereupon, the populations are classified and the populations with lower costs are selected.

Then the population with the minimum cost is selected as the good point and the random populations are generated based on this population.

Hereafter, the information, related to the power and constraints, is performed on this population and this population is transferred to the next step and then the new random populations are generated based on this population and the optimization continues until the maximum number of iterations.

\section{Simulation Results}

Application MATLAB has been used for simulation in this paper. A system with 10 generator units (24) has been selected for verifying the accuracy of above results and the results have been compared with other algorithms. Tables 1 and 2 represent the results. Results of Table 2 indicate properly the accuracy of this algorithm compared with other algorithms.

\section{Results}

In this paper, Cuckoo Search Algorithm was used for solving the UC problem. The marginal cost, calculated for programming by this algorithm in a time horizon is much lower than the methods like LR, GA, AC, and PSO especially in large-scale power systems. This algorithm easily complies with the constraints and has a good performance in the response in terms of convergence and also has the high accuracy.

\section{References}

1. Wood A F, and Wollenberg B F (1966). Power generation operation and control, $2^{\text {nd }}$ Edn., Chapter 5, New York: Wiley, 131-170.

2. Burns R M, and Gibson C A (1975). Optimization of priority lists for a unit commitment program, Proceedings of IEEE/ Power Engineering Society Summer Meeting, Paper A 75, 453-1.
3. Sheble G B (1990). Solution of the unit commitment problem by the method of unit periods, IEEE Transactions on Power Systems, vol 5, No. 1, 257-260.

4. Dillon T S, Edwin K W et al. (1978). Integer programming approach to the problem of optimal unit commitment with probabilistic reserve determination, IEEE Transactions on Power Apparatus and Systems, vol PAS-97(6), 2154-2166.

5. Garver L L (1963). Power generation scheduling by integer programming development of theory, IEEE Transactions on Power App. Systems, vol PAS-18, 730-735.

6. Snyder W L Jr., Powell H D Jr. et al. (1987). Dynamic programming approach to unit commitment, IEEE Transactions on Power Systems, vol PAS-2(2), 339350 .

7. Ouyang Z, and Shahidehpour S M (1991). An intelligent dynamic programming for unit commitment application, IEEE Transactions on Power Systems, vol 6(3), 1203-1209.

8. Muckstadt J A, and Wilson R C (1968). An application of mixed-integer programming duality to scheduling thermal generating systems, IEEE Transactions on Power Apparatus and Systems, vol PAS-87(12), 1968-1978.

9. Cohen A I, and Yoshimura M (1983). A branch-and-bound algorithm for unit commitment, IEEE Transactions on Power Apparatus and Systems, vol PAS-102(2), 444-451.

10. Merlin A, and Sandrin P (1983). A new method for unit commitment at Electricite de France, IEEE Transactions on Power Apparatus and Systems, vol PAS-102(5), 1218-1255.

11. Zhuang F, and Galiana F D (1988). Toward a more rigorous and practical unit commitment by Lagrangian relaxation, IEEE Transactions on Power Systems, vol 3(2), 763-770.

12. Ouyang Z, and Shahidehpour S M (1992). A hybrid artificial neural network/dynamic programming approach to unit commitment, IEEE Transactions on Power Systems, vol 7(1), 236-242.

13. Sasaki H, Watabable M et al. (1992). A solution method of unit commitment by artificial neural networks, IEEE Transactions on Power Systems, vol 7, No. 1, 974-985.

14. Zhuang F, and Galiana F D (1990). Unit commitment by simulated annealing, IEEE Transactions on Power Systems, vol 5(1), 311-317.

15. Dasgupta D, and McGregor D R (1994). Thermal unit commitment using genetic algorithms, IEE Proceedings Generation, Transmission and Distribution, vol 141(5), 459-465.

16. Kazarlis S A, Bakirtzis A G et al. (1996). A genetic algorithm solution to the unit commitment problem, IEEE Transactions on Power Systems, vol 11(1), 83-92. 